\title{
Consistency of breast density measured from the same women in four different MR scanners
}

\author{
Jeon-Hor Chen a) \\ Tu and Yuen Center for Functional Onco-Imaging of Department of Radiological Science, \\ University of California Irvine, California 92697-5020; Department of Radiology, China Medical \\ University Hospital, Taichung 40407, Taiwan; and Department of Medicine, School of Medicine, \\ China Medical University, Taichung 40407, Taiwan \\ Siwa Chan \\ Department of Radiology, Taichung Veterans General Hospital, Taichung 40407, Taiwan \\ Yi-Jui Liu \\ Department of Automatic Control Engineering, Feng Chia University, Taichung 40407, Taiwan \\ Dah-Cherng Yeh \\ Department of Surgery, Taichung Veterans General Hospital, Taichung 40407, Taiwan \\ Chih-Kai Chang \\ Department of Radiology, China Medical University Hospital, Taichung 40407, Taiwan \\ Li-Kuang Chen \\ Tu and Yuen Center for Functional Onco-Imaging of Department of Radiological Science, \\ University of California Irvine, California 92697-5020 \\ Wei-Fan Pan and Chih-Chen Kuo \\ Department of Radiology, China Medical University Hospital, Taichung 40407, Taiwan \\ Muqing Lin, Daniel H. E. Chang, Peter T. Fwu, and Min-Ying Su \\ Tu and Yuen Center for Functional Onco-Imaging of Department of Radiological Science, \\ University of California Irvine, California 92697-5020
}

(Received 27 February 2012; revised 18 June 2012; accepted for publication 22 June 2012; published 20 July 2012)

Purpose: To compare the breast volume (BV), fibroglandular tissue volume (FV), and percent density (PD) measured from breast MRI of the same women using four different MR scanners.

Methods: The study was performed in 34 healthy Asian volunteers using two 1.5T (GE and Siemens) and two 3T (GE and Philips) MR scanners. The BV, FV, and PD were measured on nonfat-suppressed T1-weighted images using a comprehensive computer algorithm-based segmentation method. The scanner-to-scanner measurement difference, and the coefficient of variation (CV) among the four scanners were calculated. The measurement variation between two density morphological patterns presenting as the central type and the intermingled type was separately analyzed and compared.

Results: All four scanners provided satisfactory image quality allowing for successful completion of the segmentation processes. The measured parameters between each pair of MR scanners were highly correlated, with $\mathrm{R}^{2} \geq 0.95$ for $\mathrm{BV}, \mathrm{R}^{2} \geq 0.99$ for $\mathrm{FV}$, and $\mathrm{R}^{2} \geq 0.97$ for $\mathrm{PD}$ in all comparisons. The mean percent differences between each pair of scanners were $5.9 \%-7.8 \%$ for BV, 5.3\%-6.5\% for FV, $4.3 \%-7.3 \%$ for PD; with the overall CV of $5.8 \%$ for $\mathrm{BV}, 4.8 \%$ for $\mathrm{FV}$, and $4.9 \%$ for PD. The variation of FV was smaller in the central type than in the intermingled type $(p=0.04)$.

Conclusions: The results showed that the variation of FV and PD measured from four different MR scanners is around 5\%, suggesting the parameters measured using different scanners can be used for a combined analysis in a multicenter study. (C) 2012 American Association of Physicists in Medicine. [http://dx.doi.org/10.1118/1.4736824]

Key words: fibroglandular tissue volume, percent density, breast MRI

\section{INTRODUCTION}

Many mammography studies have shown that mammographic density is a strong independent risk factor for development of breast cancer. ${ }^{1-3}$ Based on this evidence the Breast Cancer Preventive Collaboration Group has recommended that mammographic density should be considered in the risk prediction model $;{ }^{4}$ however, so far it has not been incorporated into any commonly used models. One main issue that needs to be resolved is how to obtain reliable quantitative density measures. This is a very important research area currently under rigorous investigation in the breast density research community. Due to its two-dimensional nature, mammographic density bears the intrinsic limitation of tissue overlapping, and cannot provide a true volumetric measure. Also, the measured density is susceptible to many sources of 
technical and positioning variations. ${ }^{5}$ Other methods that can measure quantitative breast density have been developed and evaluated; including volumetric measurements made based on 3D MRI. ${ }^{6-15}$ However, so far there have not been published results demonstrating the association between MRIbased density parameters and cancer risk.

Currently, breast MRI is recommended to be performed at 1.5T. The current guideline from the American Cancer Society recommends screening MRI to be performed on women with a lifetime risk greater than $20 \%$, thus only limited cases can be collected from a single site. For assessing the association between MRI-based density and cancer risk, a large dataset is required, and combining MRI from multiple centers is the only feasible way to achieve this goal. Because different scanners and different imaging protocols are used at different institutions that will lead to intrinsic differences in the acquired image quality and influence the segmented density parameters. Thus, as the first step, whether or how the densities measured from different centers can be combined needs to be investigated.

Although 3T MRI is able to provide images of higher signal-to-noise ratio and higher spatial resolution, ${ }^{16}$ the higher field inhomogeneity may compromise the image quality. ${ }^{17-19}$ Nevertheless, due to the wide availability of 3T MRI scanners, many clinical breast MRI studies are also done at 3T. The purpose of this preliminary work is to compare the measurement consistency in breast volume (BV), fibroglandular tissue volume (FV), and percent density (PD) using four different scanners, two at $1.5 \mathrm{~T}$ and two at $3 \mathrm{~T}$. The percent difference between the parameters measured by each pair of different scanners is calculated; also the overall variation among the four scanners was evaluated using the coefficient of variation. In addition, we also evaluated whether the amount of dense tissues and different breast density morphological types will affect the consistency of measurement. Based on the relative distribution pattern of fibroglandular tissue and fatty tissue, the breast is categorized as the central type (dense tissue inside surrounded by fatty tissue outside) or the intermingled type (with mixed dense tissue and fatty tissue), and the measurement variation between these two types was compared.

\section{MATERIALS AND METHODS}

\section{II.A. Subjects}

This study was approved by the Institutional Review Board and was HIPAA-compliant. All subjects provided written informed consent. Thirty-four healthy Asian female subjects (age 20-64, mean 35 year old, 26 premenopausal, and 8 postmenopausal) were recruited to receive noncontrast breast MRI studies at four different MR scanners, including GE Signa-HDx 1.5T and GE Signa-HDx 3T (GE Healthcare, Milwaukee, WI), Siemens Symphony 1.5T TIM (Siemens, Erlangen, Germany), and Philips Achieva 3.0T TX (Philips Medical Systems, Eindhoven, Netherlands). None of these 34 subjects had been diagnosed with histological-confirmed breast diseases. At the time of participation in this study they were all healthy without any breast related symptoms.
The premenopausal subjects had regular menstrual cycles and were not pregnant at the time of this study. None was taking contraceptive pills. Three postmenopausal women had a history of taking hormonal replacement therapy due to postmenopausal syndromes, but the treatment was ceased long before ( $>1$ year) participating in this study.

\section{II.B. MR imaging studies}

The four MR scans for each subject were performed at two different medical institutions in the same city, 6 miles apart, and were completed within 2 days to reduce the biological effect due to endogenous hormonal fluctuation. Prior to the study, the $\mathrm{T} 1 \mathrm{~W}$ imaging sequences for breast segmentation were tested with the assistance of clinical application scientists from each vendor to ensure the imaging quality was good enough for breast segmentation. The subjects were in prone position with arms beside the head in the GE 1.5T, GE 3T, and Philips 3T studies but positioned beside the body in the Siemens $1.5 \mathrm{~T}$ study.

In this study, the MR scans were performed bilaterally in axial sections. Since the purpose of this study was to compare the results of breast segmentation, only the 2D fast spin echo (FSE) nonfat-suppressed (or, nonfat-sat) T1 weighted images were acquired and analyzed in all four scanners; and the imaging parameters selected might not be fully comparable with the current clinical MRI standard. For GE 1.5T scanner, an eight-channel breast coil was used, with TR/TE $=607 / 9 \mathrm{~ms}$, slice thickness $=2 \mathrm{~mm}$, slice gap $=0$, phaseencoding R-L, bandwidth per pixel $=130 \mathrm{~Hz}$, field of view $(\mathrm{FOV})=380 \mathrm{~mm}$, number of signal average $=2$, and imaging matrix $=256 \times 192$. For GE 3.0T scanner, an eight-channel breast coil was used, with TR/TE $=650 / 9 \mathrm{~ms}$, slice thickness $=2 \mathrm{~mm}$, slice gap $=0$, phase encoding R-L, bandwidth per pixel $=217 \mathrm{~Hz}, \mathrm{FOV}=380 \mathrm{~mm}$, number of signal average $=2$, and imaging matrix $=256 \times 192$. For Philips 3.0T scanner, a 16-channel breast coil was used. The imaging parameters were: $\mathrm{TR} / \mathrm{TE}=645 / 9.0 \mathrm{~ms}$, parallel imaging with SENSE factor $=2$, slice thickness $=2 \mathrm{~mm}$, slice gap $=0$, phase encoding R-L, bandwidth per pixel $=174 \mathrm{~Hz}, \mathrm{FOV}=330 \mathrm{~mm}$, number of signal average $=1$, and imaging matrix $=328$ $\times$ 384. For Siemens $1.5 \mathrm{~T}$ scanner, a four-channel breast coil was used. The imaging parameters were: TR/TE $=650 / 9.8 \mathrm{~ms}$, parallel imaging with SENSE factor $=2$, slice thickness $=2 \mathrm{~mm}$, slice gap $=0$, phase encoding R-L, bandwidth per pixel $=181 \mathrm{~Hz}, \mathrm{FOV}=330 \mathrm{~mm}$, number of signal average $=1$, and imaging matrix $=330 \times 384(660$ $\times 768$ with interpolation). The imaging time of the four scanners was from 2 min $50 \mathrm{~s}$ to $4 \mathrm{~min} .8 \mathrm{~s}$.

\section{II.C. Breast and fibroglandular tissue segmentation}

The breast and fibroglandular tissue segmentation was performed using a modified published method ${ }^{14,20}$ by a research assistant (C.K.C.) with background of radiological technology and medical imaging and one year of experience in segmenting breast MR images. The four sets of T1W images of the same subject acquired using four different MR scanners 
were analyzed within two days, to ensure that the operator used the same body landmarks to segment the breast boundary and used the same standard to segment the fibroglandular tissue from the fatty tissue. The order of analysis among the four sets of images was randomly arranged from subject to subject. Before the segmentation, the operator viewed the whole axial T1W images dataset and determined the superior and inferior boundaries of the breast (the beginning and ending slices) by comparing the thickness of breast fat with the body fat. Nonbreast subcutaneous fat on the chest typically displays homogenous thickness across the chest wall, and that was used to determine where the breast starts and ends.

The breast segmentation procedures consisted of: (1) perform an initial horizontal line cut to exclude thoracic region. Depending on the morphology of the breast, a horizontal line was drawn along the posterior margin of each individual subject's sternum. If some fibroglandular tissues were chopped off, the horizontal line was lowered, up to $35 \mathrm{~mm}$ posterior from the original sternum landmark. This was to ensure that the breast region analyzed from the four scanners was consistent, and that the entire fibroglandular tissue was contained within the segmented breast. (2) Apply Fuzzy c-means (FCM) clustering ${ }^{21}$ and $b$-spline curve fitting ${ }^{10}$ to obtain the breastchest boundary. In this step, the operator checked each slice and determined if the pectoralis muscle has been removed adequately. If not, the operator had to modify the boundary manually. (3) A novel method based on nonparametric nonuniformity normalization (N3) and adaptive FCM algorithm ${ }^{20}$ was used to remove the strong intensity nonuniformity and correct the bias field for segmentation of fibroglandular tissue and fatty tissue. The N3 algorithm is a fully automatic histogram-based method, and is a popular correction method widely used in the literature. ${ }^{22}$ The N3 algorithm is able to reduce the bias field while avoiding the problem of generating erroneous contrast. (4) Apply dynamic searching to exclude the skin along the breast boundary. (5) The standard FCM algorithm is applied to classify all pixels on the image. The default setting is to use a total of six clusters, three for fibroglandular tissue, and three for fatty tissues. After completing the process, the dense tissue ROI is mapped onto the original MRI and the operator went through the images slice by slice to inspect the segmentation quality by comparing the segmented images with the original nonsegmented images. If the quality analyzed using this setting is not satisfactory, the operator can choose to use a different cluster number, typically by decreasing the total cluster number from six to five, with two or three clusters as the dense tissue. Figures 1-3 show fibroglandular tissue segmentation in three subjects.

Finally, after completing the segmentation and verification processes, a vertical line between the breasts perpendicular to the sternum was drawn to separate the left and the right breasts. Then, the quantitative BV, FV, and PD which was calculated as the ratio of $\mathrm{FV}$ over $\mathrm{BV} \times 100 \%$, were obtained. The processing time to analyze the whole set of images for both breasts of a subject, including the corrections, can be completed in $45 \mathrm{~min}$.

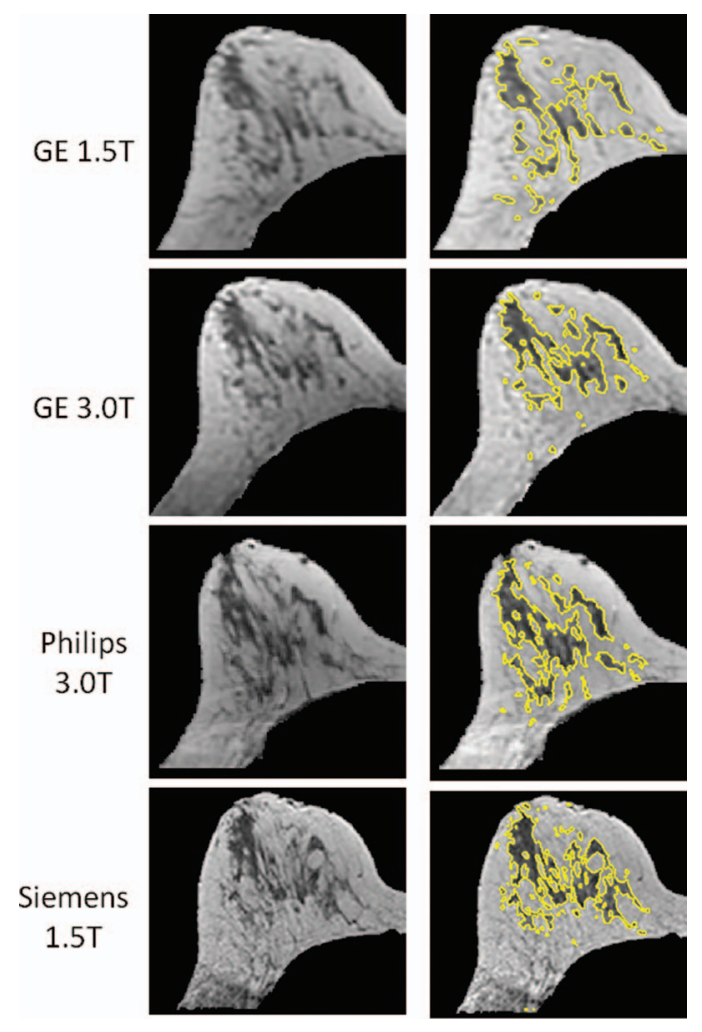

FIG. 1. A 55-year-old woman with the intermingled morphological type. (Left column) Original images; (Right column) segmented images; images from top to bottom in each column were acquired from GE 1.5T, GE 3.0T, Philips 3.0T, and Siemens 1.5T, respectively. The breast volume (BV) was $785,900,840$, and $853 \mathrm{~cm}^{3}$; the fibroglandular tissue volume (FV) was 57.0, $63.1,62.5$, and $61.8 \mathrm{~cm}^{3}$; and the percent density (PD) was $7.3 \%, 7.0 \%, 7.4 \%$, and $7.3 \%$ for GE 1.5T, GE 3.0T, Philips 3.0T, and Siemens 1.5T, respectively. The overall coefficient of variation measured from four scanners was $5.6 \%$ for $\mathrm{BV}, 4.6 \%$ for $\mathrm{FV}$, and $4.6 \%$ for PD.

\section{II.D. Classification of morphological pattern into the central type and the intermingled type}

The MR breast morphology was determined by an experienced radiologist (J.H.C.) based on the definition used in a previous study. ${ }^{23}$ Briefly, when the fibroglandular tissue of the breast was centrally located and was well surrounded by the fatty tissue, the breast was defined as a central type breast (Figs. 2 and 3). On the contrary, when the fibroglandular tissue and the fatty tissue were mixed together in the whole breast, it was defined as the intermingled breast morphology (Fig. 1). Of the 34 subjects, 17 were central type and 17 were intermingled breast morphology; therefore, there were a total of 34 central type breasts and 34 intermingled type breasts.

\section{II.E. Statistical methods}

The statistical analyses were performed using 68 breasts from 34 subjects. First, the parameters measured between each pair of MR scanners were compared. A total of six comparisons were made: namely, GE 1.5T vs GE 3.0T, GE 1.5T vs Philips 3.0T, GE 1.5T vs Siemens 1.5T, GE 3.0T vs Philips 3.0T, GE 3.0T vs Siemens 1.5T, Philips 3.0T vs Siemens 1.5T. In each paired comparison, the Pearson's correlation was used 
GE 1.5T
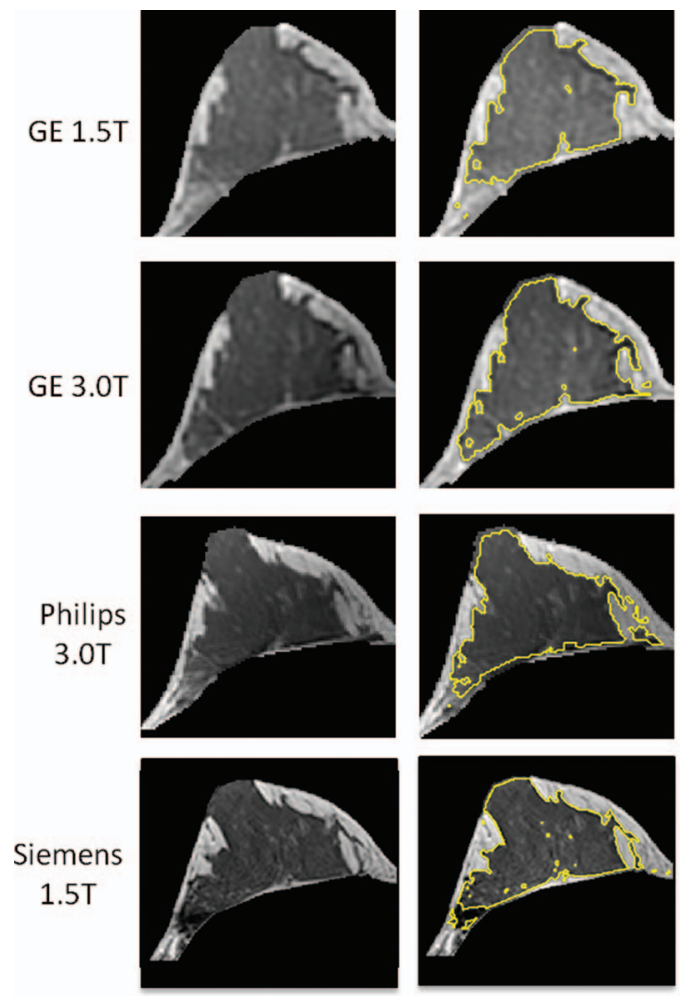

FIG. 2. A 25-year-old woman with the central morphological type. (Left column) Original images; (Right column) segmented images; images from top to bottom in each column were acquired from GE 1.5T, GE 3.0T, Philips 3.0T, and Siemens 1.5T, respectively. The breast volume (BV) was 328, 362, 390, and $366 \mathrm{~cm}^{3}$; the fibroglandular tissue volume (FV) was 148.8, 160.2, 160.5, and $158.5 \mathrm{~cm}^{3}$; and the percent density (PD) was $45.4 \%, 44.2 \%, 41.1 \%$, and $43.3 \%$ for GE 1.5T, GE 3.0T, Philips 3.0T, and Siemens 1.5T, respectively. The overall coefficient of variation measured from four scanners was $7.1 \%$ for $\mathrm{BV}, 3.5 \%$ for $\mathrm{FV}$, and $4.2 \%$ for PD.

to evaluate the overall correlation from all 68 breasts. To evaluate the level of uncertainty of the correlation between pairs of MR systems, we further analyzed the confidence interval of the correlation using the statistical analysis function (MATLAB, version 7.11.0). The data were fitted by finding the coefficient of a polynomial of degree 1 in the least-squares sense. The norm of residuals was the sum of the squares of the differences between the predicted values and the actual values, which represented the level of correlation, the smaller number the closer correlation.

For each breast, the absolute difference in the measured parameters from two scanners was calculated, and the percent difference was calculated as the absolute difference over their mean value $(\times 100 \%)$. The mean \pm standard deviation, as well as the range, of the difference calculated from all 68 breasts was evaluated. In addition, the overall consistency of the measured parameters among the four scanners was compared based on the coefficient of variation (CV), defined as the standard deviation from the four measurements divided by their mean value $(\times 100 \%)$. A higher CV indicated a larger measurement variation. The $\mathrm{CV}$ measured in the central type and the intermingled type breast was compared using the twotailed student $t$ test, using $p<0.05$ as the significance level. Lastly, in order to evaluate the relationship between the mea-

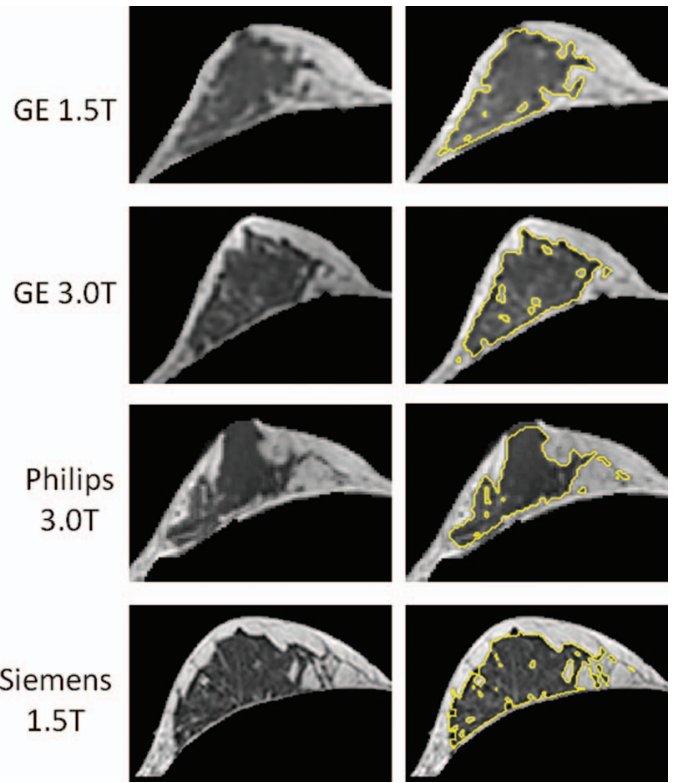

FIG. 3. A 28-year-old woman with the central morphological type. (Left column) Original images; (Right column) segmented images; images from top to bottom in each column were acquired from GE 1.5T, GE 3.0T, Philips 3.0T, and Siemens 1.5T, respectively. The breast volume (BV) was 261, 203, 275 , and $261 \mathrm{~cm}^{3}$; the fibroglandular tissue volume $(\mathrm{FV})$ was $65.5,50.8,66.5$, and $69.7 \mathrm{~cm}^{3}$; and the percent density (PD) was $25.1 \%, 25.0 \%, 24.1 \%$, and $26.7 \%$ for GE 1.5T, GE 3.0T, Philips 3.0T, and Siemens 1.5T, respectively. The overall coefficient of variation measured from four scanners was $12.8 \%$ for $\mathrm{BV}, 13.3 \%$ for $\mathrm{FV}$, and $4.3 \%$ for PD. The measurement $\mathrm{CV}$ for $\mathrm{BV}$ and $\mathrm{FV}$ of this breast is the highest among all central type cases. The high CV is caused by the much smaller BV and FV measured using the GE 3.0T scanner; however, because both the BV and the FV are smaller, the calculated PD is similar to the PD measured by the other three scanners.

surement variation with the amount of density, the 68 breasts were separated into 4 quartiles based on their FV values, 17 breasts in each quartile group, and the measurement $\mathrm{CV}$ between them was compared.

\section{RESULTS}

\section{III.A. Measurement variation between each pair of four MR scanners}

All four scanners provided satisfactory image quality for successfully completing the segmentation processes. Contrast between the fibroglandular tissue and the fatty tissue is high, as demonstrated in Figs. 1-3. Table I shows the mean value of BV, FV, and PD calculated from 68 breasts using each scanner. Figures 4-6 show the correlation of BV, FV, and PD between each pair of two MR scanners, with $\mathrm{R}^{2}=0.99$ in all six comparisons for $\mathrm{FV}$. For $\mathrm{BV}$, the $\mathrm{R}^{2}$ in six comparisons are $0.95,0.96,0.97,0.98,0.98,0.99$, which shows that the correlation was worse than that for FV. The result indicates that there is a higher variation in the segmentation of breast from the body; but as long as the fibroglandular tissue is fully contained within the segmented breast, the measurement of FV is consistent. Figure 7 shows the level of uncertainty in each pair of comparison for PD. It was obvious that the correlation was better between any pair of 1.5T GE, 3.0T GE, and 
GE1.5T vs. GE3.0T

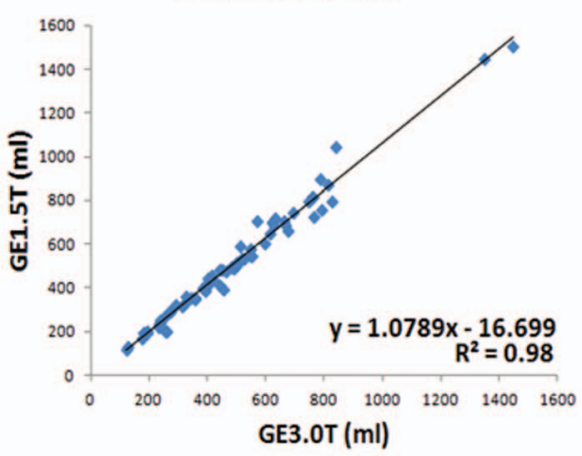

GE3.0T vs. Philips $3.0 \mathrm{~T}$

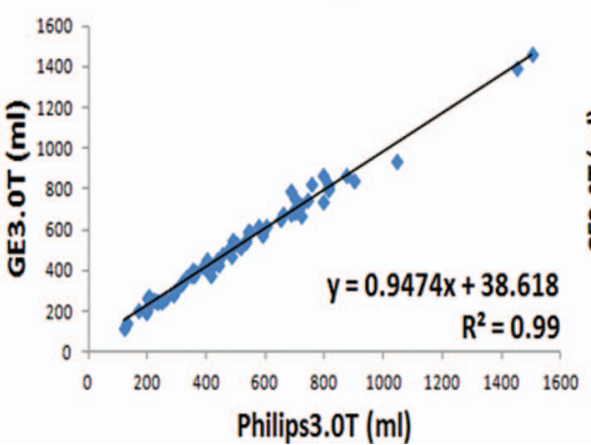

GE1.5T vs. Philips 3.0T

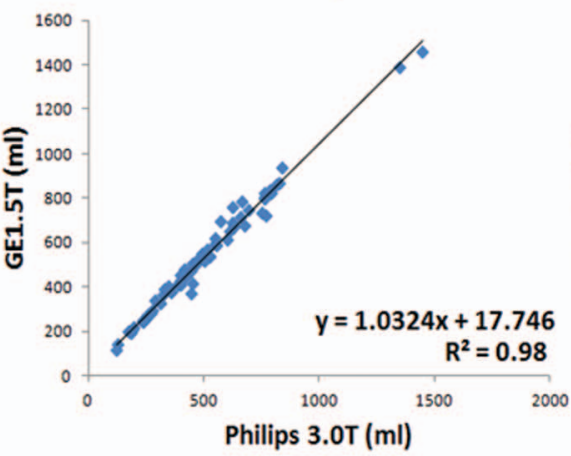

GE3.0T vs. Siemens1.5T

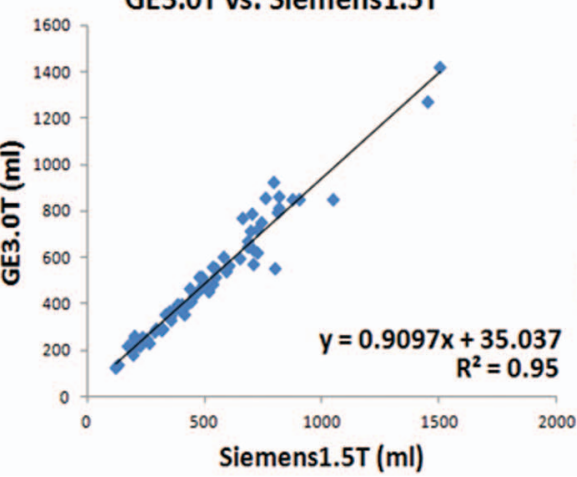

GE1.5T vs. Siemens 1.5T

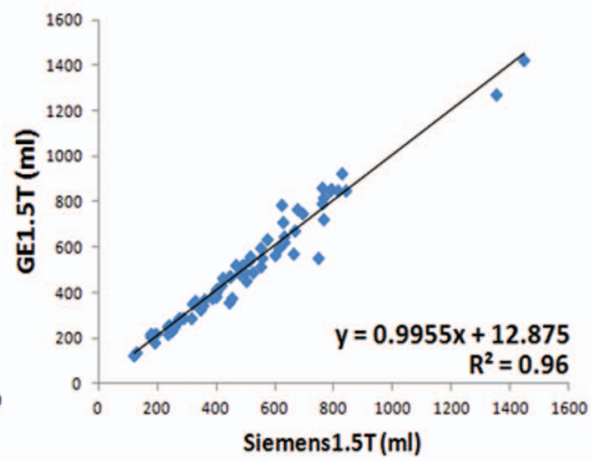

Philips 3.0T vs. Siemens1.5T

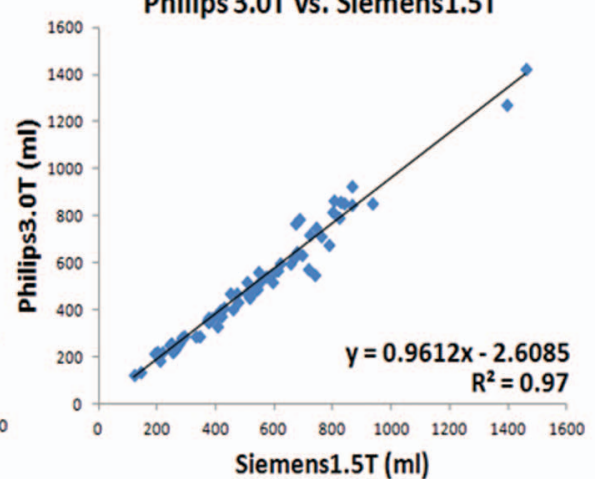

FIG. 4. The correlation of breast volume (BV) measured using each pair of different MR scanners. The BV of several subjects measured by the Siemens $1.5 \mathrm{~T}$ scanner is smaller compared to others, so in general the paired comparison with Siemens $1.5 \mathrm{~T}$ has a lower $\mathrm{R}^{2}(0.95,0.96$, and 0.97$)$ compared to the values in the other three comparisons $(0.98,0.98$, and 0.99$)$.
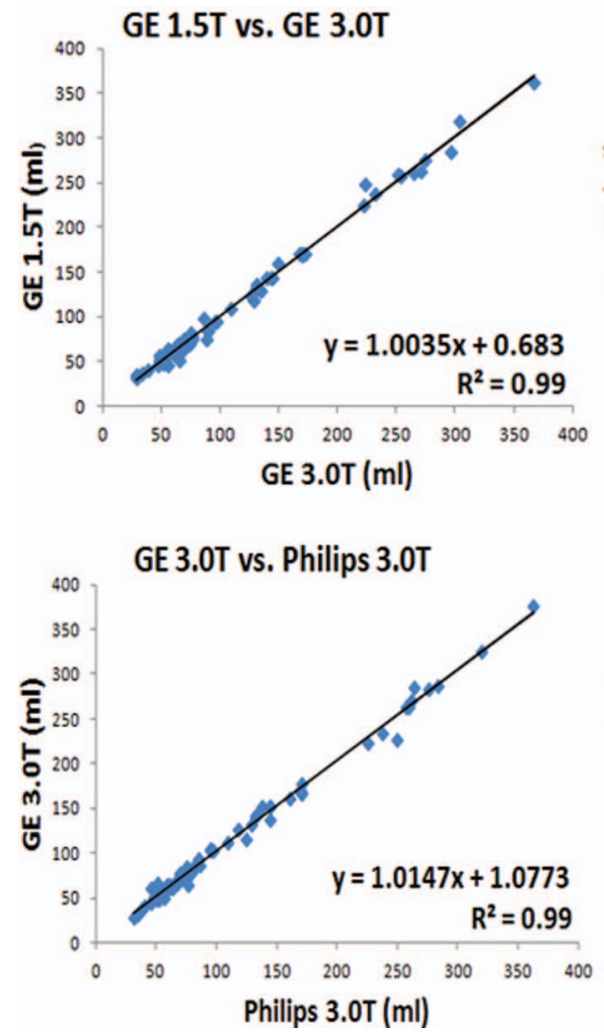
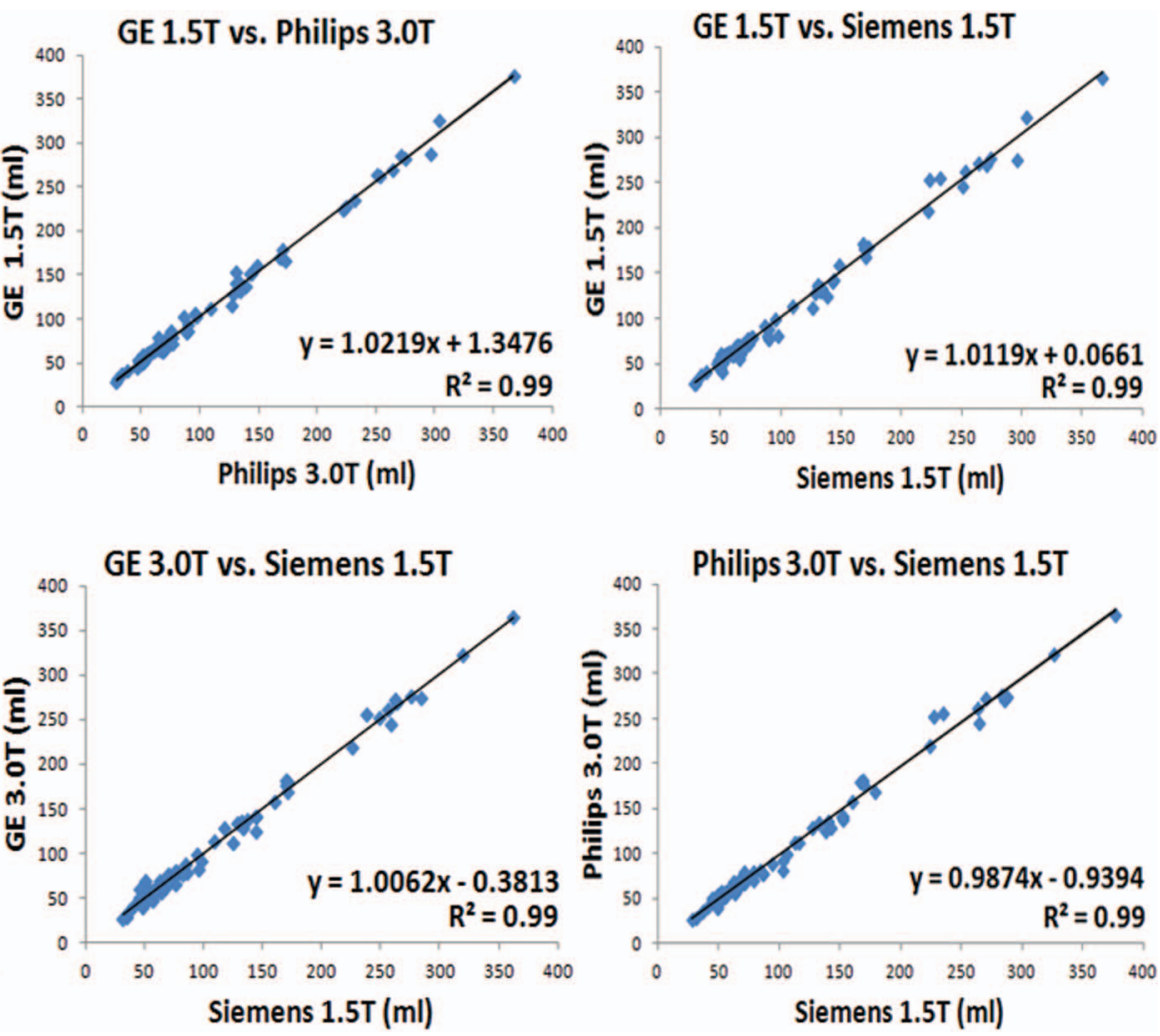

FIG. 5. The correlation of fibroglandular tissue volume (FV) measured using each pair of different MR scanners. The measured FV is highly correlated, with $\mathrm{R}^{2}=0.99$ in all six comparisons. 


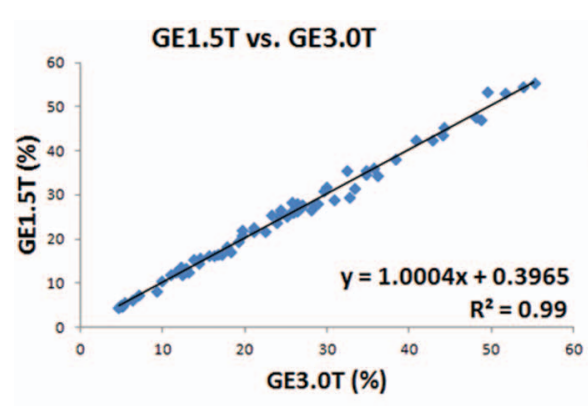

GE3.0T vs. Philips3.0T

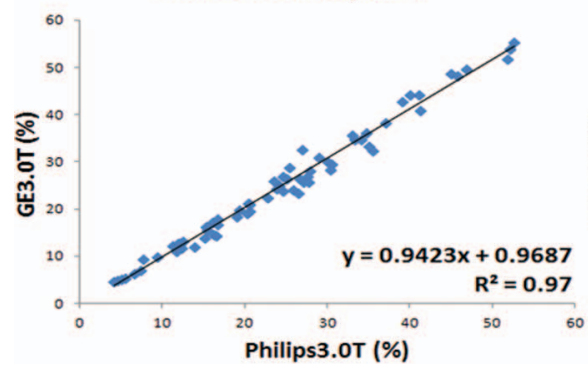

GE1.5T vs. Philips3.0T

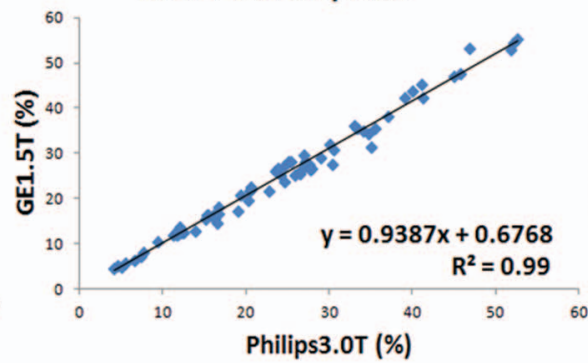

GE3.0T vs. Siemens1.5T

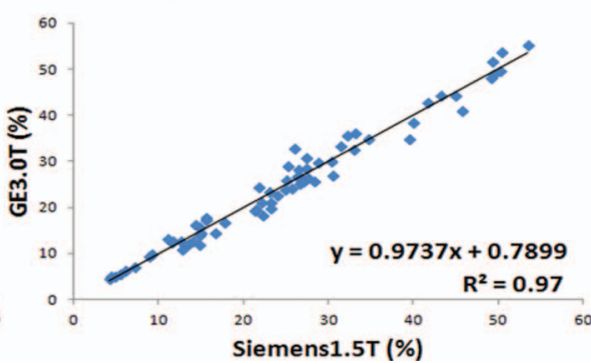

GE1.5T vs. Siemens1.5T

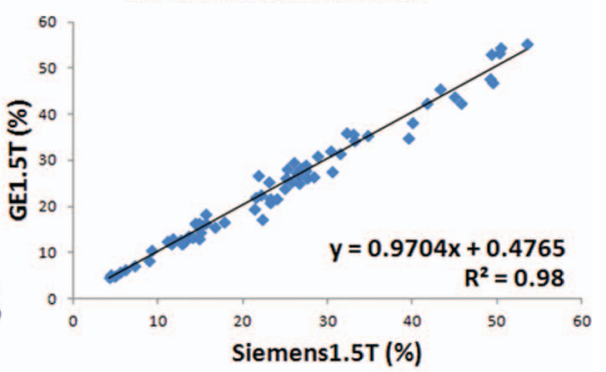

Philips3.0T vs. Siemens1.5T

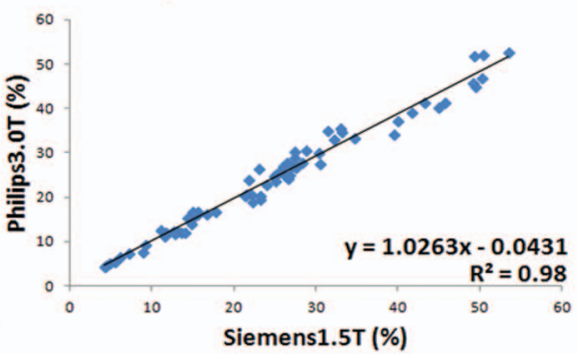

FIG. 6. The correlation of percent density (PD) measured using each pair of different MR scanners. $\mathrm{R}^{2}$ ranged from 0.97 to 0.99 .
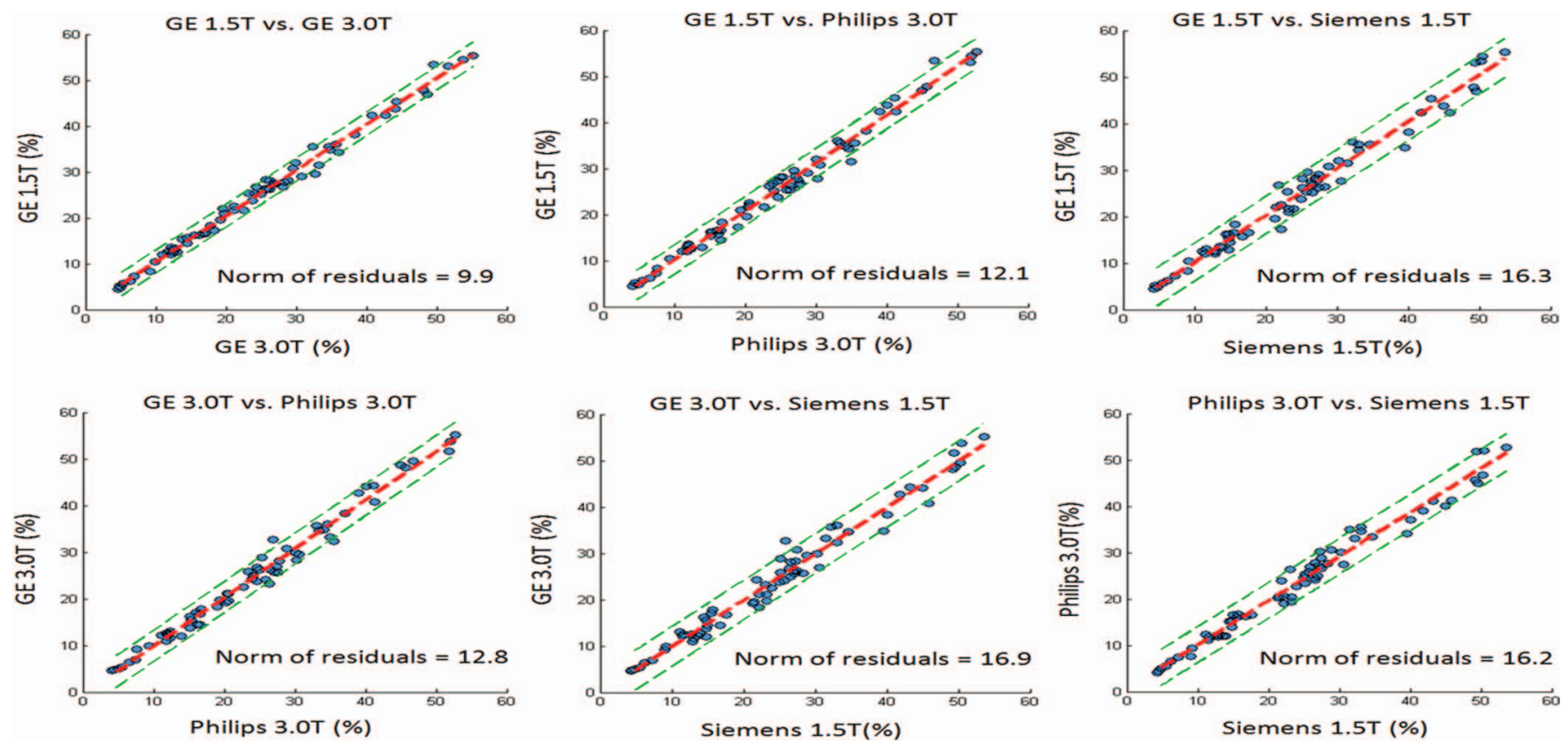

FIG. 7. The level of uncertainty in each pair of comparison for PD. The region between the two thin dashed lines contains $95 \%$ of the data. A high correlation was noted between any pair of 1.5T GE, 3.0T GE, and 3.0T Philips (all norm residuals numbers <13). When Siemens was compared with any one of the three other MR scanners, the level of uncertainty becomes bigger (norm residuals $>16$ ).

TABLE I. Mean value of BV, FV, and PD from 68 breasts measured by four MR scanners.

\begin{tabular}{lcccc}
\hline \hline & GE 1.5T & GE 3.0T & Philips 3.0T & Siemens 1.5T \\
\hline Breast volume $\left(\mathrm{cm}^{3}\right)$ & $495 \pm 253$ & $517 \pm 276$ & $528 \pm 263$ & $505 \pm 257$ \\
Fibroglandular volume $\left(\mathrm{cm}^{3}\right)$ & $114 \pm 80$ & $115 \pm 80$ & $117 \pm 82$ & $115 \pm 81$ \\
Percent density (\%) & $24.9 \pm 13.1$ & $24.5 \pm 13.1$ & $24.1 \pm 12.4$ & $24.7 \pm 12.9$ \\
\hline \hline
\end{tabular}


TABLE II. Measurement difference between each pair of two different MR scanners.

\begin{tabular}{|c|c|c|c|c|c|c|}
\hline & $\begin{array}{c}\text { GE } 1.5 \text { vs } \\
\text { GE } 3.0\end{array}$ & $\begin{array}{l}\text { GE } 1.5 \text { vs } \\
\text { Philips } 3.0\end{array}$ & $\begin{array}{c}\text { GE } 1.5 \text { vs } \\
\text { Siemens } 1.5\end{array}$ & $\begin{array}{c}\text { GE } 3.0 \text { vs } \\
\text { Philips } 3.0\end{array}$ & $\begin{array}{c}\text { GE } 3.0 \text { vs } \\
\text { Siemens } 1.5\end{array}$ & $\begin{array}{c}\text { Philips } 3.0 \text { vs } \\
\text { Siemens } 1.5\end{array}$ \\
\hline \multicolumn{7}{|c|}{ Breast volume $\left(\mathrm{cm}^{3}\right)$} \\
\hline$\Delta\left(\mathrm{cm}^{3}\right)$, mean \pm std. dev. & $34 \pm 36$ & $38 \pm 28$ & $37 \pm 37$ & $27 \pm 25$ & $41 \pm 47$ & $38 \pm 35$ \\
\hline$\Delta\left(\mathrm{cm}^{3}\right)$, range & $0.1-206$ & $0.1-135$ & $0.6-197$ & $0.3-108$ & $0.5-245$ & $1.3-186$ \\
\hline Mean percent difference & $6.2 \%$ & $7.8 \%$ & $7.4 \%$ & $5.9 \%$ & $7.8 \%$ & $7.4 \%$ \\
\hline \multicolumn{7}{|c|}{ Fibroglandular volume $\left(\mathrm{cm}^{3}\right)$} \\
\hline$\Delta\left(\mathrm{cm}^{3}\right)$, mean \pm std. dev. & $4.7 \pm 4.5$ & $5.7 \pm 4.8$ & $5.9 \pm 5.7$ & $5.3 \pm 5.0$ & $5.5 \pm 4.6$ & $6.2 \pm 5.8$ \\
\hline$\Delta\left(\mathrm{cm}^{3}\right)$, range & $0.1-25$ & $0.3-22$ & $0.5-29$ & $0.1-22$ & $0.1-20$ & $0.1-26$ \\
\hline Mean percent difference & $5.4 \%$ & $5.7 \%$ & $6.1 \%$ & $5.3 \%$ & $6.5 \%$ & $5.9 \%$ \\
\hline \multicolumn{7}{|c|}{ Percent density (\%) } \\
\hline$\Delta(\%)$, mean \pm std. dev. & $1.0 \pm 0.9$ & $1.4 \pm 1.2$ & $1.6 \pm 1.3$ & $1.3 \pm 1.2$ & $1.6 \pm 1.3$ & $1.6 \pm 1.3$ \\
\hline$\Delta(\%)$, range & $0.9-3.8$ & $1.2-6.6$ & $1.3-5.0$ & $1.2-5.7$ & $1.3-6.7$ & $1.3-5.5$ \\
\hline Mean percent difference & $4.3 \%$ & $5.9 \%$ & $6.5 \%$ & $5.8 \%$ & $7.3 \%$ & $6.4 \%$ \\
\hline
\end{tabular}

3.0T Philips (all norm residuals numbers $<13$ ) than when Siemens was compared with any one of the three other MR scanners (norm residuals $>16$ ). The scanner-to-scanner paired $t$ test, however, did not show any significant difference for any analyzed parameter. Table II shows the mean value and the range of the absolute difference in the measured $\mathrm{BV}$, $\mathrm{FV}$, and PD, as well as the mean percent difference for each paired comparison. The mean percent differences in the six paired comparisons ranged from $5.9 \%$ to $7.8 \%$ for BV, $5.3 \%$ to $6.5 \%$ for FV, $4.3 \%$ to $7.3 \%$ for PD. The ranges were comparable in all six paired comparisons, and there was not a systematic difference from any particular scanner(s).

\section{III.B. Coefficient of variation in all breasts and the central and intermingled morphological types}

The overall measurement variation from four scanners is evaluated using the $\mathrm{CV}$, listed in Table III. The $\mathrm{CV}$ was higher for BV (5.8\%) than that for FV (4.8\%) or PD (4.9\%). Among the 68 analyzed breasts, CV higher than $10 \%$ was found in BV of 5 breasts, but only in FV of 2 breasts and PD of 1 breast. In central vs intermingled type comparison, the mean $\mathrm{CV}$ for measurement of FV was $4.2 \%$ for the central type, which was significantly lower compared to $5.5 \%$ for the in-

TABLE III. The CV from four scanners in all breasts, and the central and intermingled types.

\begin{tabular}{|c|c|c|c|}
\hline & BV & $\mathrm{FV}$ & PD \\
\hline \multicolumn{4}{|c|}{ All breasts $(N=68)$} \\
\hline Mean CV & $5.8 \%$ & $4.8 \%$ & $4.9 \%$ \\
\hline Range of CV & $1.8 \%-15.2 \%$ & $1.2 \%-13.3 \%$ & $1.6 \%-11.1 \%$ \\
\hline \multicolumn{4}{|c|}{ Central type $(N=34)$} \\
\hline Mean CV & $5.5 \%$ & $4.2 \%$ & $4.7 \%$ \\
\hline Range of CV & $1.8 \%-12.8 \%$ & $1.2 \%-13.3 \%$ & $1.6 \%-11.1 \%$ \\
\hline \multicolumn{4}{|c|}{ Intermingled type $(N=34)$} \\
\hline Mean CV & $6.1 \%$ & $5.5 \%$ & $5.1 \%$ \\
\hline Range of CV & $2.3 \%-15.2 \%$ & $1.7 \%-9.6 \%$ & $2.2 \%-10.4 \%$ \\
\hline
\end{tabular}

termingled type $(p=0.04)$. The mean $\mathrm{CV}$ for $\mathrm{BV}$ was $5.5 \%$ for the central type and $6.1 \%$ for the intermingled type, not significantly different $(p=0.3)$. The mean $\mathrm{CV}$ for PD was $4.7 \%$ for the central type and $5.1 \%$ for the intermingled type, not significant either $(p=0.3)$.

\section{III.C. Coefficient of variation in four quartile groups based on FV values}

The 68 breasts were separated into 4 quartiles based on their FV values, with 17 breasts in each group. The mean value and the range of FV were: $48.2(28.9-59.9) \mathrm{cm}^{3}$ in the first quartile; $66.9(60.4-75.7) \mathrm{cm}^{3}$ in the second quartile; $110.7(78.4 \sim 144.9) \mathrm{cm}^{3}$ in the third quartile; and 234.9 $(145.8 \sim 368) \mathrm{cm}^{3}$ in the fourth quartile. The coefficient of variation for $\mathrm{FV}$ was $2.9 \%$ in the fourth quartile group, which was significantly smaller compared to the $5.5 \%$ in the first quartile ( $p=0.001), 5.0 \%$ in the second quartile $(p=0.008)$, and $5.0 \%$ in the third quartile $(p=0.005)$. The smaller CV in the fourth quartile was due to their much higher mean FV value, so the results did not suggest an obvious trend of measurement variation related to different amount of fibroglandular tissues.

\section{DISCUSSION}

Quantitative 3D MR-based analysis of breast density can potentially provide an imaging biomarker for assessing cancer risk or predicting therapeutic efficacy of hormonal treatments. MRI provides detailed 3D distribution of fibroglandular tissue not subject to the tissue-overlapping problem as in mammography, and thus is suitable for volumetric measurements. We have developed a comprehensive computer algorithm-based segmentation method for quantitative analysis of whole BV and FV on 3D MRI, ${ }^{14,20}$ which has shown both intra- and interoperator variation smaller than $5 \%$. The method has been applied to study the age- and race-related differences, ${ }^{24}$ as well as the change in patients receiving chemotherapy ${ }^{25}$ and tamoxifen. ${ }^{26}$ The variation coming from the operator(s) is one 
major concern, which can be solved by minimizing the interventions that need to be provided by the operator. During the past several years we have been continuing to improve the robustness of method by standardizing the processes. For segmentation of the breast, using easily recognizable body landmarks such as sternum can minimize the judgment call from different operators. For the segmentation of fibroglandular tissue, one important step is to implement a powerful bias field correction method that can work in a large imaging field, and we have shown that the $\mathrm{N} 3+\mathrm{FCM}$ and the coherent local intensity clustering (CLIC) algorithm both worked very well. ${ }^{20}$ Then, within a homogeneous intensity field, it is possible to fix the number of FCM clusters used for segmentation of fibroglandular tissue. We have evaluated several different combinations, and decided to choose a total of six clusters as the default setting, three for fibroglandular, and three for fatty tissues. Before MRI-based breast density can be used as a reliable imaging biomarker, it is necessary to thoroughly evaluate the dependence of the measured parameters on possible biological and technical factors. In addition to evaluating operator variations, we have recently reported the biological fluctuation of the measured density associated with the change of endogenous hormone during a menstrual cycle. ${ }^{27}$ In another study, we investigated the variation of parameters analyzed based on fat-sat and nonfat-sat images. ${ }^{28}$

In the present study, we set out to investigate another very important technical factor that may affect the measured density parameter-the use of different MR scanners. This is particularly important for combining data obtained from different performance sites that use different MR scanners. In order to facilitate such a multicenter study, the consistency of data measured from different sites should be verified. ${ }^{29}$ The optimal design is to have the same subject receive repeated examinations using different scanners. The Alzheimer's Disease NeuroImaging Initiative (ADNI) (Refs. 30-32) has diligently arranged "traveling volunteers" to receive brain MRI using optimized imaging protocol across all sites. ${ }^{32}$ Similarly, in this study we recruited 34 healthy women to receive breast MRI study using four different MR scanners located at two nearby medical institutions in the same city. In order to minimize the biological variations such as that coming from the endogenous hormone, the four studies were completed within two days.

We chose to use four scanners from three major manufacturers, GE 1.5T and GE 3.0T, Siemens 1.5T, and Philips 3.0T. These scanners were equipped with the state-of-the-art breast coil and imaging sequences. The nonfat-sat T1-weighted images acquired using FSE pulse sequence were used for the analysis. This sequence was chosen because of the high image quality and a good tissue contrast between the fibroglandular tissue and the fatty tissue. The quality of MR images depends on the homogeneity of the B0 field, the B1 transmit field of the body RF coil, and the receiver profile of the surface breast coil. 3T MR offers a higher signal-to-noise ratio than $1.5 \mathrm{~T},{ }^{16}$ but it comes with technical problems that may offset the benefits. B0 field is usually homogeneous in most magnets and does not contribute much to signal variations on T1 FSE images without fat-sat. B1 field may lead to spatial variations of the delivered radiofrequency pulses (or, nonuniform flip angle) in a large FOV, which then affects signal intensity. ${ }^{33}$ The variation of the $\mathrm{B} 1$ transmission is higher at 3T compared to 1.5T. Compared with the B0 field strengths and inhomogeneity and the B1 transmission inhomogeneity, the different receiver surface breast coil is probably the biggest source of variations. The types of coils, manufacturers, models, and number of elements will affect the quality of breast MR images. The strong bias field presents a difficulty for thresholding- or clustering-based segmentation approaches. Although we have shown that our N3+FCM method ${ }^{20}$ worked well, sometimes a local contrast adjustment is still needed, and there is room for further improvements. Along the same line of research, Kruggel et al. reported the difference in the segmented gray matter and white matter in a large ADNI brain MRI dataset acquired using 17 different MR scanner models from all three major manufacturers. ${ }^{34}$ Despite the standardized protocol, differences across scanners were considerable. It was concluded that two most likely factors contributing to the different segmentation results are scannerdependent geometrical inaccuracies and differences in the tissue contrast. ${ }^{34}$

In this study, the pulse sequences were tested with the assistance of clinical application scientists from each vendor to ensure satisfactory imaging quality prior to the MR examinations. The measurement variation $(\mathrm{CV})$ of $\mathrm{BV}, \mathrm{FV}$, and PD among the four different scanners was 5.8\%, 4.8\%, and $4.9 \%$, respectively, which fell in the range of operator variation and positional difference of approximately $5 \%$ using the same scanner. ${ }^{14}$

For measurement of $\mathrm{BV}$, we used the sternum as the body landmark for determining the posterior boundary of the breast. Although this was easily identifiable, some fibroglandular tissue extending into the axilla might be chopped off. This problem was commonly encountered in our cohort of Asian women who have relatively dense breasts. If this happened, the operator needed to lower the horizontal cutting line 5-35 $\mathrm{mm}$ posterior to the sternum to ensure that all fibroglandular tissue was included in the segmented breast. When a shift was used, the same shift was used in the analyses of this subject's four datasets, so the same criteria were applied. Ensuring that all fibroglandular tissue was contained within the segmented breast helped in minimizing the measurement variation of FV and PD (CV from four scanners $4.8 \%$ and $4.9 \%$, respectively). The $\mathrm{CV}$ for $\mathrm{BV}(5.8 \%)$ was slightly higher compared to FV and PD. Also, among the 68 analyzed breasts, CV higher than $10 \%$ was found in BV of 5 breasts but only occurred in FV of 2 breasts and PD of 1 breast. A higher variation in BV was likely due to the uncertainty in the determination of the beginning and ending slices. This may change with different body and arm positioning, and thus cannot be fixed. Since the breast tissue in the starting and ending slices is mainly fatty tissue, this uncertainty will not affect the measurement of $\mathrm{FV}$ as much. In the five breasts showing $\mathrm{CV}$ of BV higher than $10 \%$, it was mainly because the BV measured by one scanner was much different compared to the other three scanners. In the case shown in Fig. 3, the BV of both breasts measured by GE 3.0T was smaller compared to the 
others, and in another subject the BV of both breasts measured by Siemens $1.5 \mathrm{~T}$ was much smaller. However, the difference was not consistently coming from one particular scanner, thus there was not a noticeable systematic difference in the measurement of BV. For the correlation of PD between each pair of MR scanners, the level of uncertainty became more obvious when the Siemens scanner was compared with any one of the three other scanners (Fig. 7). It was postulated that the difference of arm position (arms beside the head in the GE 1.5T, GE 3T, and Philips 3T studies, but arms beside the body in the Siemens $1.5 \mathrm{~T}$ study) may partially account for the results.

The variation in the measurement of FV was more likely coming from the different tissue contrast and the partial volume effect, which was associated with the density morphological types and the spatial resolution. In this study, the spatial resolution along the read-out direction of the Philips 3.0T and Siemens $1.5 \mathrm{~T}$ (acquisition matrix $328 \times 384$ and $330 \times 384$ ) was higher than that of the GE 1.5T and GE 3.0T (acquisition matrix $256 \times 192$ ), but we did not observe systematic differences in the measured parameters between these scanners. We further investigated whether different breast morphologies would affect the measurement consistency, and found that the central type breast tended to have more consistent measurement of the FV than the intermingled type $(p=0.04)$. As seen in the three case examples shown in Figs. 1-3, the partial volume effect is clearly noted in the intermingled type; and in contrast, the signal intensity is more homogeneous in the central type. Nonetheless, the measurement variation for the intermingled type can be managed well by using a consistent FCM clustering method, as seen in the case shown in Fig. 1 that has the overall measurement $\mathrm{CV}$ of $5.6 \%$ for BV, $4.6 \%$ for $\mathrm{FV}$, and $4.6 \%$ for PD. We also evaluated the variation in breasts with different amount of fibroglandular tissues. A lower CV was found in the fourth quartile group with the highest FV volume, which was very likely coming from its high mean value that makes the CV small. Therefore, we did not observe a trend of measurement variation with respect to the amount of fibroglandular tissues. We have inspected the results from all 68 breasts using 4 scanners, and as the results shown in Table II, the ranges of CV in all six paired comparisons were comparable, and there was not a systematic trend related to a specific scanner that could explain the high variations observed in some subjects. Therefore, the degree of variations presented in this study is very likely representing the natural variations coming from both the technical and the biological sources that will be encountered in a typical breast MRI study.

This study had several limitations. The sample size was small. The subjects were Asian women with small and dense breasts, thus the results may not be generalized to other populations. Specifically, for women who have very fatty breasts with only scarce fibroglandular tissue, the measurement of FV may be more severely affected by the partial volume effect, and the percent variation will be higher due to the small denominator. Unfortunately in this study, we did not include women with very fatty breast to investigate its effect. We only had eight postmenopausal women, and they still had relatively dense breasts. Variations of the different scanners, including the scanners per se, the sequences/scan parameters used, and the gradient nonlinearity, can directly affect the quality of the breast segmentation. The variation of matrix choices among scanners in this study might introduce some unnecessary variation in segmented volume results. For the 2 GE scanners, with a FOV of $38 \mathrm{~cm}$ and $256 \times 192$ matrix, the spatial resolution is less than that clinically acceptable for ACR Breast MRI Accreditation. Our previous study, ${ }^{28}$ with a matrix size of $480 \times 480 \mathrm{~mm}$ and FOV $=31-38 \mathrm{~cm}$, however, has shown that FV measured on downsampled images $(240 \times 240)$ only showed a small $(<5 \%)$ difference.

\section{CONCLUSION}

The results from this pilot study show that when a welldeveloped segmentation method is used, consistent density parameters from the same women can be obtained from images acquired using different MR scanners. It was found that the overall variation was around 5\% for the measurements of FV and PD, suggesting that breast density data analyzed from different scanners in multiple centers may be used for a combined analysis.

\section{ACKNOWLEDGMENT}

This work was supported in part by National Institutes of Health (NIH)/National Cancer Institute (NCI) Grant Nos. R01 CA127927 and R03 CA136071.

\footnotetext{
a) Author to whom correspondence should be addressed. Electronic mail: jeonhc@uci.edu; Telephone: 949-824-9327; Fax: 949-824-3481.

${ }^{1}$ N. F. Boyd, H. Guo, L. J. Martin, L. Sun, J. Stone, E. Fishell, R. A. Jong, G. Hislop, A. Chiarelli, S. Minkin, and M. J. Yaffe, "Mammographic density and the risk and detection of breast cancer," N. Engl. J. Med. 356, 227-236 (2007)

${ }^{2}$ V. A. McCormack and I. dos Santos Silva, "Breast density and parenchymal patterns as markers of breast cancer risk: A meta-analysis," Cancer Epidemiol. Biomarkers Prev. 15, 1159-1169 (2006).

${ }^{3}$ C. M. Vachon, V. S. Pankratz, C. G. Scott, S. D. Maloney, K. Ghosh, K. R. Brandt, T. Milanese, M. J. Carston, and T. A. Sellers, "Longitudinal trends in mammographic percent density and breast cancer risk," Cancer Epidemiol. Biomarkers Prev. 16, 921-928 (2007).

${ }^{4}$ R. J. Santen, N. F. Boyd, R. T. Chlebowski, S. Cummings, J. Cuzick, M. Dowsett, D. Easton, J. F. Forbes, T. Key, S. E. Hankinson, A. Howell, J. Ingle, and Breast Cancer Prevention Collaborative Group, "Critical assessment of new risk factors for breast cancer: Considerations for development of an improved risk prediction model," Endocr. Relat. Cancer 14, 169-187 (2007), review.

${ }^{5}$ J. A. Harvey and V. E. Bovbjerg, "Quantitative assessment of mammographic breast density: Relationship with breast cancer risk," Radiology 230, 29-41 (2004).

${ }^{6}$ J. Eng-Wong, J. Orzano-Birgani, C. K. Chow, D. Venzon, J. Yao, C. E. Galbo, J. A. Zujewski, and S. Prindiville, "Effect of Raloxifene on mammographic density and breast magnetic resonance imaging in premenopausal women at increased risk for breast cancer," Cancer Epidemiol. Biomarkers Prev. 17, 1696-1701 (2008).

${ }^{7}$ M. Khazen, R. Warren, C. Boggis, E. C. Bryant, S. Reed, I. Warsi, L. J. Pointon, G. E. Kwan-Lim, D. Thompson, R. Eeles, D. Easton, D. G. Evans, M. O. Leach, and Collaborators in the United Kingdom Medical Research Council Magnetic Resonance Imaging in Breast Screening (MARIBS) Study, "A pilot study of compositional analysis of the breast and estimation of breast mammographic density using three-dimensional T1-weighted magnetic resonance imaging," Cancer Epidemiol. Biomarkers Prev. 17, 2268-2274 (2008).
} 
${ }^{8}$ J. Wei, H. P. Chan, M. A. Helvie, M. A. Roubidoux, B. Sahiner, L. M. Hadjiiski, C. Zhou, S. Paquerault, T. Chenevert, and M. M. Goodsitt, "Correlation between mammographic density and volumetric fibroglandular tissue estimated on breast MR images," Med. Phys. 31, 923-942 (2004).

${ }^{9}$ S. van Engeland, P. R. Snoeren, H. Huisman, C. Boetes, and N. Karssemeijer, "Volumetric breast density estimation from full-field digital mammograms," IEEE Trans. Med. Imaging 25, 273-282 (2006).

${ }^{10}$ N. A. Lee, H. Rusinek, J. Weinreb, R. Chandra, H. Toth, C. Singer, and G. Newstead, "Fatty and fibroglandular tissue volumes in the breasts of women 20-83 years old: Comparison of $\mathrm{x}$-ray mammography and computer-assisted MR imaging," AJR, Am. J. Roentgenol. 168, 501-506 (1997).

${ }^{11}$ J. Yao, J. A. Zujewski, J. Orzano, S. Prindiville, and C. Chow, "Classification and calculation of breast fibroglandular tissue volume on SPGR fat suppressed MRI,” Med. Imaging Proc. SPIE 5747, 1942-1949 (2005).

${ }^{12}$ C. Klifa, J. Carballido-Gamio, L. Wilmes, A. Laprie, C. Lobo, E. Demicco, M. Watkins, J. Shepherd, J. Gibbs, and N. Hylton, "Quantification of breast tissue index from MR data using fuzzy cluster," Proc. IEEE Eng. Med. Biol. Soc. 3, 1667-1670 (2004).

${ }^{13}$ C. Klifa, J. Carballido-Gamio, L. Wilmes, A. Laprie, J. Shepherd, J. Gibbs, B. Fan, S. Noworolski, and N. Hylton, "Magnetic resonance imaging for secondary assessment of breast density in a high-risk cohort," Magn. Reson. Imaging 28, 8-15 (2010).

${ }^{14}$ K. Nie, J. H. Chen, S. Chan, M. K. Chau, H. J. Yu, S. Bahri, T. Tseng, O. Nalcioglu, and M. Y. Su, "Development of a quantitative method for analysis of breast density based on three-dimensional breast MRI," Med. Phys. 35, 5253-5262 (2008).

${ }^{15}$ D. J. Thompson, M. O. Leach, G. Kwan-Lim, S. A. Gayther, S. J. Ramus, I. Warsi, F. Lennard, M. Khazen, E. Bryant, S. Reed, C. R. Boggis, D. G. Evans, R. A. Eeles, D. F. Easton, R. M. Warren, and UK study of MRI screening for breast cancer in women at high risk (MARIBS), "Assessing the usefulness of a novel MRI-based breast density estimation algorithm in a cohort of women at high genetic risk of breast cancer: The UK MARIBS study," Breast Cancer Res. Treat. 11, R80 (2009).

${ }^{16}$ R. Rakow-Penner, B. Daniel, H. Yu, A. Sawyer-Glover, and G. H. Glover, "Relaxation times of breast tissue at 1.5T and 3T measured using IDEAL," J Magn Reson Imaging 23, 87-91 (2006).

${ }^{17}$ C. K. Kuhl, H. Kooijman, J. Gieseke, and H. H. Schild, "Effect of B1 inhomogeneity on breast MR imaging at 3.0 T," Radiology 244, 929-930 (2007).

${ }^{18}$ R. M. Mann, C. K. Kuhl, K. Kinkel, and C. Boetes, "Breast MRI: Guidelines from the European Society of Breast Imaging," Eur. Radiol. 18, 13071318 (2008).

${ }^{19}$ C. A. Azlan, P. D. Giovanni, T. S. Ahearn, S. K. Semple, F. J. Gilbert, and T. W. Redpath, "B1 transmission-field inhomogeneity and enhancement ratio errors in dynamic contrast-enhanced MRI (DCE-MRI) of the breast at 3T,” J. Magn. Reson. Imaging 31, 234-239 (2010).

${ }^{20}$ M. Lin, S. Chan, J. H. Chen, D. Chang, K. Nie, S. T. Chen, C. J. Lin, T. C. Shih, O. Nalcioglu, and M. Y. Su, "A new bias field correction method combining N3 and FCM for improved segmentation of breast density on MRI," Med. Phys. 38, 5-14 (2011).

${ }^{21}$ W. Chen, M. L. Giger, and U. Bick, "A fuzzy c-means (FCM)-based approach for computerized segmentation of breast lesions in dynamic contrast-enhanced MR images," Acad Radiol. 13, 63-72 (2006).

${ }^{22}$ J. G. Sled, A. P. Zijdenbos, and A. C. Evans, "A nonparametric method for automatic correction of intensity nonuniformity in MRI data," IEEE Trans. Med. Imaging 17, 87-97 (1998).

${ }^{23}$ K. Nie, J. H. Chen, D. Chang, C. C. Hsu, O. Nalcioglu, and M. Y. Su, "Quantitative analysis of breast parenchymal patterns using 3D fibrog- landular tissue segmentation based on MRI," Med. Phys. 37, 217-226 (2010).

${ }^{24}$ K. Nie, M. Y. Su, M. K. Chau, S. Chan, H. Nguyen, T. Tseng, Y. Huang, C. E. McLaren, O. Nalcioglu, and J. H. Chen, "Age- and race-dependence of the fibroglandular breast density analyzed on 3D MRI," Med. Phys. 37, 2770-2776 (2010)

${ }^{25}$ J. H. Chen, K. Nie, S. Bahri, C. C. Hsu, F. T. Hsu, H. N. Shih, M. Lin, O. Nalcioglu, and M. Y. Su, "MRI evaluation of decrease of breast density in the contralateral normal breast of patients receiving neoadjuvant chemotherapy," Radiology 255, 44-52 (2010).

${ }^{26}$ J. H. Chen, Y. C. Chang, D. Chang, Y. T. Wang, K. Nie, R. F. Chang, O. Nalcioglu, C. S. Huang, and M. Y. Su, "Reduction of breast density following tamoxifen treatment evaluated by 3-D MRI: Preliminary study," Magn. Reson. Imaging 29, 91-98 (2011).

${ }^{27}$ S. Chan, M. Y. Su, F. J. Lei, J. P. Wu, M. Lin, O. Nalcioglu, S. A. Feig, and J. H. Chen, "Menstrual cycle related fluctuations in breast density measured," Radiology 261, 744-751 (2011).

${ }^{28}$ D. H. E. Chang, J. H. Chen, M. Lin, S. Bahri, H. J. Yu, R. S. Mehta, K. Nie, D. J. Hsiang, O. Nalcioglu, and M. Y. Su, "Comparison of breast density measured on MR images acquired using fat-suppressed versus nonfat-suppressed sequences," Med. Phys. 38, 5961-5968 (2011).

${ }^{29}$ A. J. Buckler, L. Bresolin, N. R. Dunnick, D. C. Sullivan, H. J. Aerts, B. Bendriem, C. Bendtsen, R. Boellaard, J. M. Boone, P. E. Cole, J. J. Conklin, G. S. Dorfman, P. S. Douglas, W. Eidsaunet, C. Elsinger, R. A. Frank, C. Gatsonis, M. L. Giger, S. N. Gupta, D. Gustafson, O. S. Hoekstra, E. F. Jackson, L. Karam, G. J. Kelloff, P. E. Kinahan, G. McLennan, C. G. Miller, P. D. Mozley, K. E. Muller, R. Patt, D. Raunig, M. Rosen, H. Rupani, L. H. Schwartz, B. A. Siegel, A. G. Sorensen, R. L. Wahl, J. C. Waterton, W. Wolf, G. Zahlmann, and B. Zimmerman, "Quantitative imaging test approval and biomarker qualification: Interrelated but distinct activities," Radiology 259, 875-884 (2011).

${ }^{30}$ X. Hua, A. D. Leow, S. Lee, A. D. Klunder, A. W. Toga, N. Lepore, Y. Y. Chou, C. Brun, M. C. Chiang, M. Barysheva, C. R. Jack, Jr., M. A. Bernstein, P. J. Britson, C. P. Ward, J. L. Whitwell, B. Borowski, A. S. Fleisher, N. C. Fox, R. G. Boyes, J. Barnes, D. Harvey, J. Kornak, N. Schuff, L. Boreta, G. E. Alexander, M. W. Weiner, P. M. Thompson, and Alzheimer's Disease Neuroimaging Initiative, "3D characterization of brain atrophy in Alzheimer's disease and mild cognitive impairment using tensor-based morphometry," Neuroimage 41, 19-34 (2008)

${ }^{31}$ X. Hua, A. D. Leow, N. Parikshak, S. Lee, M. C. Chiang, A. W. Toga, C. R. Jack, Jr., M. W. Weiner, P. M. Thompson, and Alzheimer's Disease Neuroimaging Initiative, "Tensor-based morphometry as a neuroimaging biomarker for Alzheimer's disease: An MRI study of $676 \mathrm{AD}$, MCI, and normal subjects," Neuroimage 43, 458-469 (2008).

${ }^{32}$ C. R. Jack, Jr., M. A. Bernstein, N. C. Fox, P. Thompson, G. Alexander, D. Harvey, B. Borowski, P. J. Britson, J. L. Whitwell, C. Ward, A. M. Dale, J. P. Felmlee, J. L. Gunter, D. L. Hill, R. Killiany, N. Schuff, S. FoxBosetti, C. Lin, C. Studholme, C. S. DeCarli, G. Krueger, H. A. Ward, G. J. Metzger, K. T. Scott, R. Mallozzi, D. Blezek, J. Levy, J. P. Debbins, A. S. Fleisher, M. Albert, R. Green, G. Bartzokis, G. Glover, J. Mugler, and M. W. Weiner, "The Alzheimer's disease neuroimaging initiative (ADNI): MRI methods," J. Magn. Reson. Imaging 27, 685-691 (2008).

${ }^{33}$ C. E. Mountford, P. Stanwell, and R. Saadallah, "Breast MR Imaging at 3.0T," Radiology 248, 319-320 (2008).

${ }^{34}$ F. Kruggel, J. Turner, T. L. Muftuler, and The Alzheimer's Disease Neuroimaging Initiative, "Impact of scanner hardware and imaging protocol on image quality and compartment volume precision in the ADNI cohort," Neuroimage 49, 2123-2133 (2010). 